\title{
NLTE model calculations for the solar atmosphere with an iterative treatment of opacity distribution functions ${ }^{\star}$
}

\author{
M. Haberreiter ${ }^{1, \star \star}$, W. Schmutz ${ }^{1}$, and I. Hubeny ${ }^{2}$ \\ 1 Physikalisch-Meteorologisches Observatorium Davos, World Radiation Center, Dorfstrasse 33, 7260 Davos Dorf, Switzerland \\ e-mail: [margit.haberreiter; werner.schmutz] @pmodwrc.ch \\ 2 Steward Observatory, University of Arizona, Tucson, AZ 85721, USA \\ e-mail: hubeny@as.arizona.edu
}

Received 8 February 2008 / Accepted 17 October 2008

\begin{abstract}
Context. Modeling the variability of the solar spectral irradiance is a key factor in understanding the Sun's influence on the climate of the Earth.

Aims. As a first step toward calculating the solar spectral irradiance variations, we reproduce the solar spectrum for the quiet Sun over a broad wavelength range with an emphasis on the UV.

Methods. We introduce the radiative transfer code COSI, which calculates solar synthetic spectra under conditions of non-local thermodynamic equilibrium (NLTE). A self-consistent simultaneous solution of the radiative transfer and the statistical equation for the level populations guarantees that the correct physics is considered for wavelength regions where the assumption of local thermodynamic equilibrium (LTE) breaks down. The new concept of iterated opacity distribution functions (NLTE-ODFs) is presented, through which all line opacities are included in the NLTE radiative transfer calculation.

Results. We show that it is essential to include the line opacities in the radiative transfer to reproduce the solar spectrum in the UV. Conclusions. Through the implemented scheme of NLTE-ODFs, the COSI code is successful in reproducing the spectral energy distribution of the quiet Sun.
\end{abstract}

Key words. radiative transfer - Sun: atmosphere - Sun: chromosphere - Sun: UV radiation - line: profiles - atomic data

\section{Introduction}

While it cannot be the cause of the climate change over the past 20 years (Lockwood \& Fröhlich 2007), the total solar irradiance (TSI) is known to have influenced the Earth's pre-industrial climate (Labitzke 2005; Kilifarska \& Haigh 2005; Shindell et al. 2003). However, it has not yet been fully identified how exactly the irradiance variations affect the climate. It has been argued that the direct forcing of the varying TSI is too small to produce the observed climate changes, such as those observed during the little ice age (Shindell et al. 2001). One of the prime candidates for an indirect effect is the variation in the UV part of the solar spectrum, which varies much more than the total irradiance. In particular it has already been shown that H I $121.6 \mathrm{~nm}$ (Lyman- $\alpha$ ) and the Herzberg band around $200 \mathrm{~nm}$ affect ozone and the temperature in the stratosphere (Rozanov et al. 2006, 2002; Egorova et al. 2004).

To understand the solar spectrum variations, it is essential to consider all the relevant physical processes in the formation of the solar spectrum. This research has already been carried out by several authors, who use different approaches and focus on various wavelength ranges. Calculations of solar spectra in 1D over broad spectral bands were carried out by Kurucz $(1991,2005)$ based on LTE radiative transfer calculations with the ATLAS9 and ATLAS12 code in plane-parallel

\footnotetext{
* Table 2 is only available in electronic form at http: //www. aanda.org

$\star \star$ Present affiliation: LASP, University of Colorado, 1234 Innovation Drive, Boulder, CO, 80303, USA.
}

symmetry. Moreover, Vernazza et al. (1981), Allende Prieto et al. (2003a,b), Fontenla et al. (1999, 2007), and Avrett \& Loeser (2008) have all calculated solar spectra in NLTE and planeparallel symmetry for the visible and IR. Improved understanding of the solar spectrum formation has been achieved by the detailed study of individual spectral lines by Ayres et al. (2006) and Collet et al. (2005), who calculated models out of local thermodynamic equilibrium (NLTE). Additionally, Hubeny \& Lites (1995), Uitenbroek (2001, 2002), Fox et al. (2004), and Fontenla et al. $(1999,2007)$ have improved the synthetic calculations by including partial redistribution (PRD). Finally, Short \& Hauschildt (2005) computed model spectra of cool stars in NLTE and spherical symmetry. Furthermore, 3D NLTE radiative transfer calculations based on 3D hydrodynamic simulations have been carried out, e.g. by Asplund et al. (2000), Koesterke et al. (2008). In this paper we limit ourselves to radiative transfer calculations in $1 \mathrm{D}$, as our reconstruction approach of the spectral solar irradiance is based on 1D model atmosphere structures of solar surface features and their distribution on the solar surface (Wenzler et al. 2006, 2005; Haberreiter et al. 2005; Krivova \& Solanki 2005).

The formation of the solar spectrum is, in some spectral ranges, dominated by an immense number of spectral lines, e.g. the iron-like elements in the UV, also known as UV line haze. Due to the characteristics of the solar temperature structure there are two effects of the line opacity, which depend on the height of the line formation, that must be distinguished. One effect is the line blocking in the photosphere leading to a decrease in intensity. The other effect is the excess of intensity due to emission 
lines in the chromosphere. Because of NLTE effects, i.e. the illumination from above, the chromospheric emission lines can lead to overionization at lower layers. In this paper we do not focus on the chromospheric emission lines apart from Lyman$\alpha$, as they can only be correctly calculated if all atomic processes are treated in full NLTE but discuss the line blocking in the photosphere.

As shown by Collet et al. (2005), the lack of photospheric line blocking leads to an incorrect excess of ionization. The authors find differences between calculations including and excluding line opacity in NLTE calculations with a modified version of the statistical equilibrium code MULTI (Carlsson 1986) for cool stars with solar metallicity. They show that the inclusion of sampled background line opacities decreases the mean intensity field at a given depth and thereby reduces the radiative ionization rates. The authors find that the decrease in ionization in turn changes the line strength and leads to a Fe abundance that is $0.1-0.15$ dex higher than in calculations without line blocking. From this it follows that all opacities contributing to the line blocking have to be accounted for in NLTE radiative transfer calculations.

There have already been a number of approaches for including line opacities in the background continuum opacity. Anderson \& Athay (1989) and Anderson (1989) first introduced the concept of super levels that allows for numerous levels of certain atoms with similar energies, which are assumed to be in LTE with respect to each other. This approach was later adopted by Dreizler \& Werner (1993) and Hubeny \& Lanz (1995), who used ODFs for the transitions between the superlevels. Describing line opacities with a Monte-Carlo evaluation has been applied by Schmutz et al. (1991), Schaerer \& Schmutz (1994), Schmutz (1997), and de Marco \& Schmutz (1999) in the calculation of the mass loss of hot stars. Short \& Hauschildt (2005) include more than 100000 spectral lines or iron-like ions that blanket the UV-band self-consistently in the NLTE calculation. Collet et al. (2005) and Avrett \& Loeser (2008) include the line opacities in the background continuum opacity via sampled line opacities. Our approach improves the self-consistency of the NLTE calculation by including the line opacities from all known transitions of all ions in the radiative transfer calculations by means of iterated opacity distribution functions which include some NLTE effects, and therefore, we name these functions NLTE-ODFs.

The main purpose of this paper is not so much to provide a detailed analysis of line profiles, but to demonstrate the calculation of realistic solar energy distribution over a broad wavelength range with an emphasis on the UV and to be able to reproduce the solar observations and reference spectra over a broad wavelength range.

In the next section we describe the radiative transfer code COSI, the model atoms, the model atmosphere, the implemented cross sections and the different sets of solar abundances used in our calculations. Then, in Sect. 3 we validate the performance of COSI in LTE against the ATLAS12 calculations. In Sect. 4 we describe the new concept of NLTE-ODFs and present the NLTE calculations carried out with COSI. In Sect. 5 the results are discussed and finally in Sect. 6 the conclusions are presented.

\section{2. $\cos I$}

\subsection{Introduction}

COSI (COde for Solar Irradiance) is a combination of two codes, i.e. a model atmosphere code, developed by Hamann \& Schmutz (1987) and Schmutz et al. (1989), hereafter COSIMA, and the spectrum synthesis program SYNSPEC, going back to Hubeny (1981) and further developed by Hubeny (1988) and Hubeny \& Lanz (1995). The new method introduced by this paper is the use of NLTE-ODFs for including the effects of line blanketing replacing the Monte-Carlo evaluation of the line opacity that was used in earlier applications (Schmutz et al. 1991; Schaerer \& Schmutz 1994; Schmutz 1997; de Marco \& Schmutz 1999). COSIMA calculates the NLTE populations for a set of specified atomic levels by solving the radiative transport equations simultaneously with the equations for statistical equilibrium. The radiative transfer is solved in spherical symmetry (Mihalas 1978; Peraiah 2001), which yields a more reliable emerging intensity at the limb than a plane-parallel geometry and allows to calculate line of sights at and beyond the solar limb (Haberreiter et al. 2008), the contribution of which becomes increasingly important for UV/EUV wavelengths which are formed higher in the solar atmosphere.

The spectral synthesis code takes the NLTE level populations for the explicit levels from COSIMA and assumes LTE populations for the non-explicit atomic levels. The latter are calculated in LTE relative to the NLTE ground states. Population numbers of lines that connect a lower explicit NLTE level and a non-explicit upper level are assumed to be in LTE with respect to the explicit level. Partition functions are used for the calculation of the ionization equilibria in LTE, following the Saha-Equation. The partition functions for the elements $\mathrm{H}$ to $\mathrm{Zn}$ are taken from Traving et al. (1966), and for the heavier elements the coefficients for polynomial fits are taken from Irwin (1981). This allows us to considers all bound-bound transitions from hydrogen to thallium, leading to more than $2.8 \times 10^{6}$ lines. The linelist was provided by Kurucz (2006).

\subsection{Atmosphere structures}

For the validation of COSI in LTE we apply the atmosphere structure for the quiet Sun by Kurucz (1991, hereafter ASUN), which is calculated in LTE and has a monotonic outward decreasing temperature profile with the outer boundary at the temperature minimum.

The NLTE spectra are calculated with the semi-empirical structure of the solar atmosphere for average supergranule cell interior (Model C) by Fontenla et al. (1999), further referred to as F1999.

\subsection{Model atoms}

The atomic model of hydrogen consists of negative hydrogen, 10 levels of neutral hydrogen each representing the quantum numbers up to $n=10$, and the ion. Helium is modeled by 10 levels of neutral helium and one level for the ground state of singly ionized helium. Furthermore, we have implemented all metals up to zinc in the NLTE calculation. Currently we account for a total number 61 ions and 114 explicit NLTE levels as given in Table 2. The metal levels are generally selected according to their importance for the solar UV continuum opacity, e.g. their ionization edges are in the wavelength range from 100 to $400 \mathrm{~nm}$.

All bound-bound and bound-free transitions connecting the explicit levels are fully included in the NLTE calculation. In the present implementation the millions of additional transitions are set to LTE relative to the ground level of the corresponding ionization stage, which is allowed to deviate from LTE and explicitly calculated. Thus the LTE transitions are to some 
extent affected by NLTE conditions and in turn determine the NLTE-ODFs.

For line transitions connecting an explicit lower level with a non-explicit upper level, the latter is calculated in LTE relative to the NLTE population of the lower level. Here however, an inaccuracy occurs for the chromospheric lines that are calculated in LTE, because this leads to an overestimation of the line emissivity. To avoid this excess of emission in the spectral synthesis the line emissivity for each depth point outward of the temperature minimum is calculated with the excitation temperature set to the value of the temperature minimum, i.e. as $\epsilon_{L, \lambda}=B_{\lambda, T_{\min }} \kappa_{L, \lambda}$, with $B$ being the Planck function and $L$ the depth point index of the atmosphere structure. We are aware of the fact that this approach clearly underestimates the chromospheric emission lines. However, the large excess of the emission lines due to their wrong LTE calculation is not the reasonable solution either. This shortcoming will be addressed in the future by including all the relevant levels in the NLTE calculation.

\subsection{Cross sections}

\subsubsection{Radiative processes}

The bound-free and free-free radiative processes involving negative hydrogen are implemented according to John (1988). The photoionization cross section for the ground state of hydrogen is calculated according to Mihalas (1967) and the cross sections of the other hydrogen levels are adopted from Seaton (1960). The bound-free radiative processes for helium are calculated according to Koester et al. (1985). Free-free cross sections of positively charged ions and electrons are calculated with an hydrogenic approximation using the cross sections by Berger (1956) and Karzas \& Latter (1961).

Table 2 gives the sources for the photoionization cross sections for all the 114 explicit NLTE-levels accounted for in COSI. For the ions $\mathrm{CI}, \mathrm{Na}$ I, Mg I, Al I, Si I, S I and Ca I the photoionization cross sections are taken from the Opacity Project (Seaton et al. 1994), and the ones for Fe I are provided by Bautista (1997) and Bautista \& Pradhan (1997). We linearly interpolate the complex photoionization cross sections to the slightly coarser frequency grid employed in COSI (Haberreiter et al. 2003). As an example, the cross section of $\mathrm{Mg} \mathrm{I}$ is given in Fig. 1. As theoretical ionization energies are slightly off from the observed ones, we corrected the theoretical values to agree with the measured energies taken from the National Institute of Standard and Technology (NIST) Atomic Spectra Database. In addition, Thomson scattering and Rayleigh scattering are included in COSI.

\subsubsection{Collisional processes}

In COSI the collisional processes involving negative hydrogen include the collisional detachment by electrons and neutral hydrogen and charge neutralization with protons as given by Lambert \& Pagel (1968), Eq. (2b,c,e) therein. The collisional bound-bound cross sections of neutral hydrogen, the optically permitted transitions of helium, as well as of the neutral and ionized metals are calculated using the formula of Jefferies (1968), p. 118, Eq. (6.24). Collisional processes of neutral hydrogen, other than with negative hydrogen, are not yet included.

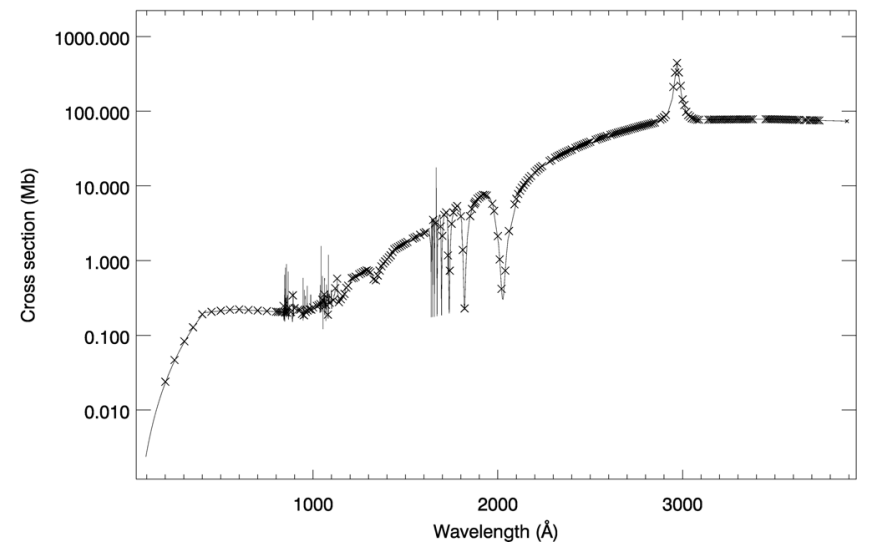

Fig. 1. Photoionization cross section for the third level of neutral $\mathrm{Mg}$ (MgI 3 in Table 2) implemented in COSI. The solid line is the original data from the Opacity Project and the crosses are the data interpolated to the frequency grid applied in COSI.

Table 1. List of applied solar abundances by number relative to $H$ and their ratio for the elements (El) with atomic number $A$ and atomic mass $m_{\mathrm{A}}$.

\begin{tabular}{lrrlll}
\hline \hline $\mathrm{El}$ & $A$ & \multicolumn{1}{c}{$m_{\mathrm{A}}$} & \multicolumn{1}{c}{$\mathrm{K} 91$} & \multicolumn{1}{c}{$\mathrm{F} 2007$} & $\frac{\mathrm{F} 2007}{\mathrm{~K} 91}$ \\
\hline $\mathrm{H}$ & 1 & 1.008 & $1.000 \mathrm{E} 0$ & $1.000 \mathrm{E} 0$ & 1.000 \\
$\mathrm{He}$ & 2 & 4.003 & $9.769 \mathrm{E}-2$ & $1.000 \mathrm{E}-1$ & 1.024 \\
$\mathrm{Li}$ & 3 & 6.941 & $1.447 \mathrm{E}-11$ & $1.122 \mathrm{E}-11$ & 0.775 \\
$\mathrm{Be}$ & 4 & 9.012 & $1.414 \mathrm{E}-11$ & $2.399 \mathrm{E}-11$ & 1.696 \\
$\mathrm{~B}$ & 5 & 10.810 & $3.985 \mathrm{E}-10$ & $5.012 \mathrm{E}-10$ & 1.258 \\
$\mathrm{C}$ & 6 & 12.011 & $3.635 \mathrm{E}-4$ & $2.455 \mathrm{E}-4$ & 0.675 \\
$\mathrm{~N}$ & 7 & 14.007 & $1.123 \mathrm{E}-4$ & $6.026 \mathrm{E}-5$ & 0.536 \\
$\mathrm{O}$ & 8 & 16.000 & $8.521 \mathrm{E}-4$ & $4.571 \mathrm{E}-4$ & 0.536 \\
$\mathrm{~F}$ & 9 & 18.918 & $3.634 \mathrm{E}-8$ & $3.631 \mathrm{E}-8$ & 0.999 \\
$\mathrm{Ne}$ & 10 & 20.179 & $1.231 \mathrm{E}-4$ & $6.981 \mathrm{E}-5$ & 0.562 \\
$\mathrm{Na}$ & 11 & 22.990 & $2.140 \mathrm{E}-6$ & $1.479 \mathrm{E}-6$ & 0.691 \\
$\mathrm{Mg}$ & 12 & 24.305 & $3.806 \mathrm{E}-5$ & $3.388 \mathrm{E}-5$ & 0.890 \\
$\mathrm{Al}$ & 13 & 26.982 & $2.954 \mathrm{E}-6$ & $2.344 \mathrm{E}-6$ & 0.793 \\
$\mathrm{Si}$ & 14 & 28.086 & $3.552 \mathrm{E}-5$ & $3.236 \mathrm{E}-5$ & 0.911 \\
$\mathrm{P}$ & 15 & 30.974 & $2.822 \mathrm{E}-7$ & $2.291 \mathrm{E}-7$ & 0.812 \\
$\mathrm{~S}$ & 16 & 32.060 & $1.624 \mathrm{E}-5$ & $6.918 \mathrm{E}-6$ & 0.426 \\
$\mathrm{Cl}$ & 17 & 35.453 & $3.166 \mathrm{E}-7$ & $3.162 \mathrm{E}-7$ & 0.999 \\
$\mathrm{Ar}$ & 18 & 39.948 & $3.635 \mathrm{E}-6$ & $1.514 \mathrm{E}-6$ & 0.417 \\
$\mathrm{~K}$ & 19 & 39.098 & $1.320 \mathrm{E}-7$ & $1.202 \mathrm{E}-7$ & 0.911 \\
$\mathrm{Ca}$ & 20 & 40.080 & $2.293 \mathrm{E}-6$ & $2.042 \mathrm{E}-6$ & 0.890 \\
$\mathrm{Sc}$ & 21 & 44.956 & $1.260 \mathrm{E}-9$ & $1.122 \mathrm{E}-9$ & 0.890 \\
$\mathrm{Ti}$ & 22 & 47.900 & $9.783 \mathrm{E}-8$ & $7.943 \mathrm{E}-8$ & 0.812 \\
$\mathrm{~V}$ & 23 & 50.941 & $1.001 \mathrm{E}-8$ & $1.000 \mathrm{E}-8$ & 0.999 \\
$\mathrm{Cr}$ & 24 & 51.996 & $4.683 \mathrm{E}-7$ & $4.365 \mathrm{E}-7$ & 0.932 \\
$\mathrm{Mn}$ & 25 & 54.938 & $2.457 \mathrm{E}-7$ & $2.455 \mathrm{E}-7$ & 0.999 \\
$\mathrm{Fe}$ & 26 & 55.847 & $4.683 \mathrm{E}-5$ & $2.818 \mathrm{E}-5$ & 0.602 \\
$\mathrm{Co}$ & 27 & 58.933 & $8.327 \mathrm{E}-8$ & $8.318 \mathrm{E}-8$ & 0.999 \\
$\mathrm{Ni}$ & 28 & 58.700 & $1.780 \mathrm{E}-6$ & $1.698 \mathrm{E}-6$ & 0.954 \\
$\mathrm{Cu}$ & 29 & 63.546 & $1.624 \mathrm{E}-8$ & $1.622 \mathrm{E}-8$ & 0.999 \\
$\mathrm{Zn}$ & 30 & 65.380 & $3.985 \mathrm{E}-8$ & $3.304 \mathrm{E}-8$ & 0.999 \\
\hline
\end{tabular}

\subsection{Solar abundances}

The study of solar abundances is an ongoing research topic and various data sets are used by different authors. For the synthetic spectra presented in this paper, the abundances used by Kurucz (1991, K91) were applied in the LTE COSI calculations using the atmosphere structure ASUN. The abundances used by Fontenla et al. (2007, F2007), which are based on Asplund et al. (2005), are employed for the calculations using the F1999 atmosphere structure. Table 1 lists both abundance sets and their ratio 

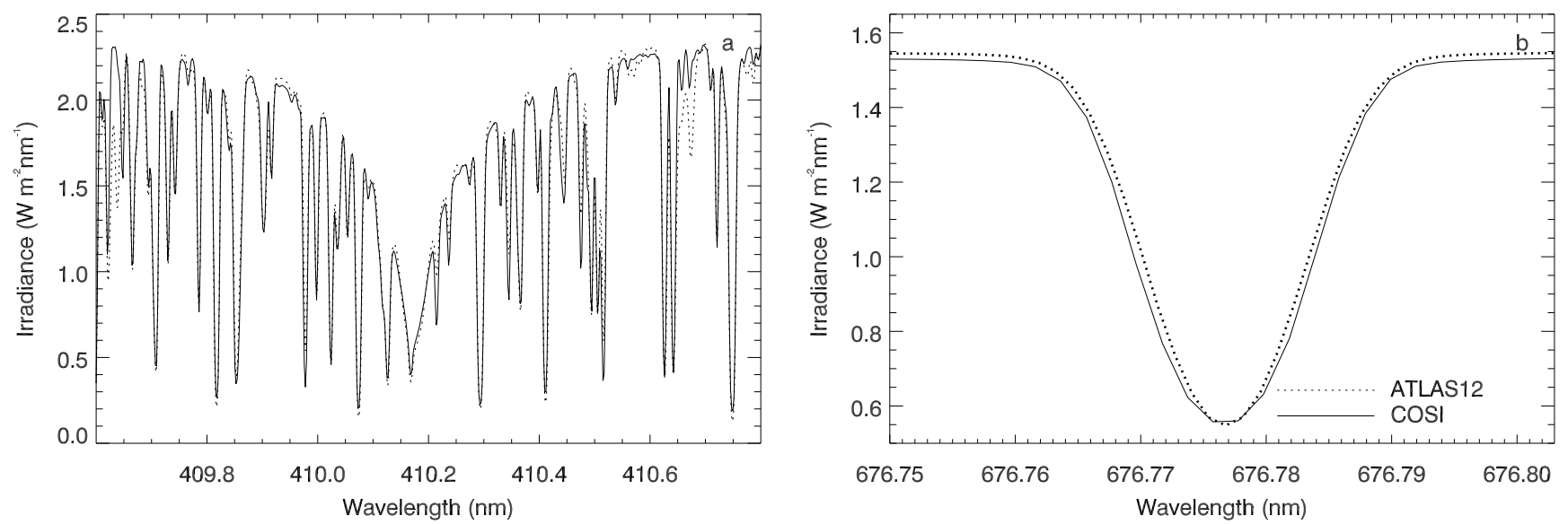

Fig. 2. Comparison of ATLAS12 calculation by Kurucz (2005) (dotted line) and COSI calculation (solid line) for H I $410.1 \mathrm{~nm}$ (panel a)) and $\mathrm{Ni}$ I $676.8 \mathrm{~nm}$ (panel b)), using the same abundances, atmosphere structure, microturbulence $\left(1.5 \mathrm{~km} \mathrm{~s}^{-1}\right)$, Gaussian macroturbulence $(F W H M=$ $\left.1.5 \mathrm{~km} \mathrm{~s}^{-1}\right)$ and rotation broadening $\left(2 \mathrm{~km} \mathrm{~s}^{-1}\right)$.

for the elements implemented in the atomic model. K91 is used in the COSI LTE calculations. F2007 is the abundance used in all the NLTE calculations presented in this paper.

\section{Code validation in LTE}

In order to validate the overall performance of COSI in LTE we calculated the emergent flux of the quiet Sun with COSI, applying the same atmosphere structure (ASUN), abundances (K91), and values for microturbulence $\left(1.5 \mathrm{~km} \mathrm{~s}^{-1}\right)$, macroturbulence (Gaussian $F W H M=1.5 \mathrm{~km} \mathrm{~s}^{-1}$ ), and rotation broadening $\left(2 \mathrm{~km} \mathrm{~s}^{-1}\right)$ as Kurucz (2005) for the ATLAS12 calculations. The rotation broadening has been implemented as given by Gray (1992). Figure 2 shows the calculations with ATLAS12 carried out by Kurucz (2005) (dotted line) and COSI (solid line) for the spectrum centered at H I $410.1 \mathrm{~nm}$ (panel a) and Ni I $\lambda 676.8 \mathrm{~nm}$ (panel b). The line profiles of H I $410.1 \mathrm{~nm}$ and in particular the wings of this line are almost identical, showing that in COSI the radiative transfer and the Stark broadening are computed consistently with the ATLAS12 code. The absolute flux level of the continuum at the Ni I wavelength is slightly less than in the ATLAS12 calculation. As a result, the line profile calculated with COSI appears slightly broader than the ATLAS12 calculation. However, the profiles are practically identical when related to the same continuum level.

The same calculation over the wavelength range from 200 to $1000 \mathrm{~nm}$ is shown in Fig. 3. In panel (a) the continuum irradiance spectrum calculated with ATLAS12 (dotted line) and with COSI (solid line) are shown, where in the latter the hydrogen lines are included. The difference shortward of the Balmer jump at $364.5 \mathrm{~nm}$ is mainly due to the photoionization cross section of the third Mg I level, shown in Fig. 1, apparently not accounted for in the ATLAS12 calculation. Panel (b) shows the corresponding synthetic spectra including all spectral lines. To allow a better comparison the high resolution line spectra have been convolved with a 1-nm boxcar filter. Generally, the calculations show a good agreement, and the overall shape of the continuum spectrum is well reproduced. The strongest discrepancies are between $\sim 350$ and $430 \mathrm{~nm}$. The differences between the spectra are due to missing molecular lines in COSI and due to different photoionization cross sections as evident from panel (a). In Fig. 3 panel (c), the ratio between the COSI and ATLAS12 line spectra is given for each 1-nm bin. Below $420 \mathrm{~nm}$ the ratio indicates some deviation while for longer wavelengths the ratio is very close to unity.

To further illustrate the COSI to ATLAS12 comparison, Fig. 5 shows the same continuum calculation as in Fig. 3a, but over a narrower wavelength range in the UV. The discrepancy between 210 and $250 \mathrm{~nm}$ (panel a) can be explained by different photoionization cross sections which lead to a higher continuum flux in the COSI calculation than in the ATLAS 12 calculation. We tested the effect of increasing the Mg I 2 photoionization cross section by a factor of 2 in COSI. This leads to a much better agreement between both calculations but is inconsistent with Opacity Project data. The lower continuum calculated with COSI between 290 and $300 \mathrm{~nm}$ is a consequence of a resonance in the photoionization cross section of Mg I 3 level (cf. Fig. 1). However, this resonance is not confirmed by the observations taken with the Solar Stellar Irradiance Comparison Experiment (SOLSTICE) (Rottman et al. 1993; Woods et al. 1993), as the measurements agree better with the ATLAS12 calculations. This indicates that there is still a need for improved atomic data. Nevertheless, from 260 to $280 \mathrm{~nm}$ (panel a) the continua give comparable results. In Fig. 6 the LTE line spectra calculated with COSI are validated against the SOLSTICE measurements. The fairly good agreement indicates that the spectrum synthesis with COSI in LTE is calculated reasonably well. However, from $~ 310$ to $320 \mathrm{~nm}$ COSI leads to a considerable higher irradiance than ATLAS12 and the SOLSTICE observation. The missing molecular lines of the $\mathrm{CN}$-band explain the discrepancy between $~ 385$ and $390 \mathrm{~nm}$.

\section{NLTE calculations}

\subsection{NLTE-ODFS}

\subsubsection{Theory}

For a complete calculation of the radiative transfer all relevant processes, e.g. the bound-bound, bound-free, and free-free processes have to be taken into account. Due to the huge number of bound-bound transitions the opacity of all spectral lines is considerable and must be taken into account in the NLTE radiative transfer calculation. In COSI only the line transitions between the explicit levels given in Table 2 are calculated in NLTE. Thus, the line opacity of a large number of lines is not directly included in the solution of the NLTE radiative transport. Indirectly we account for them by applying opacity distribution functions 

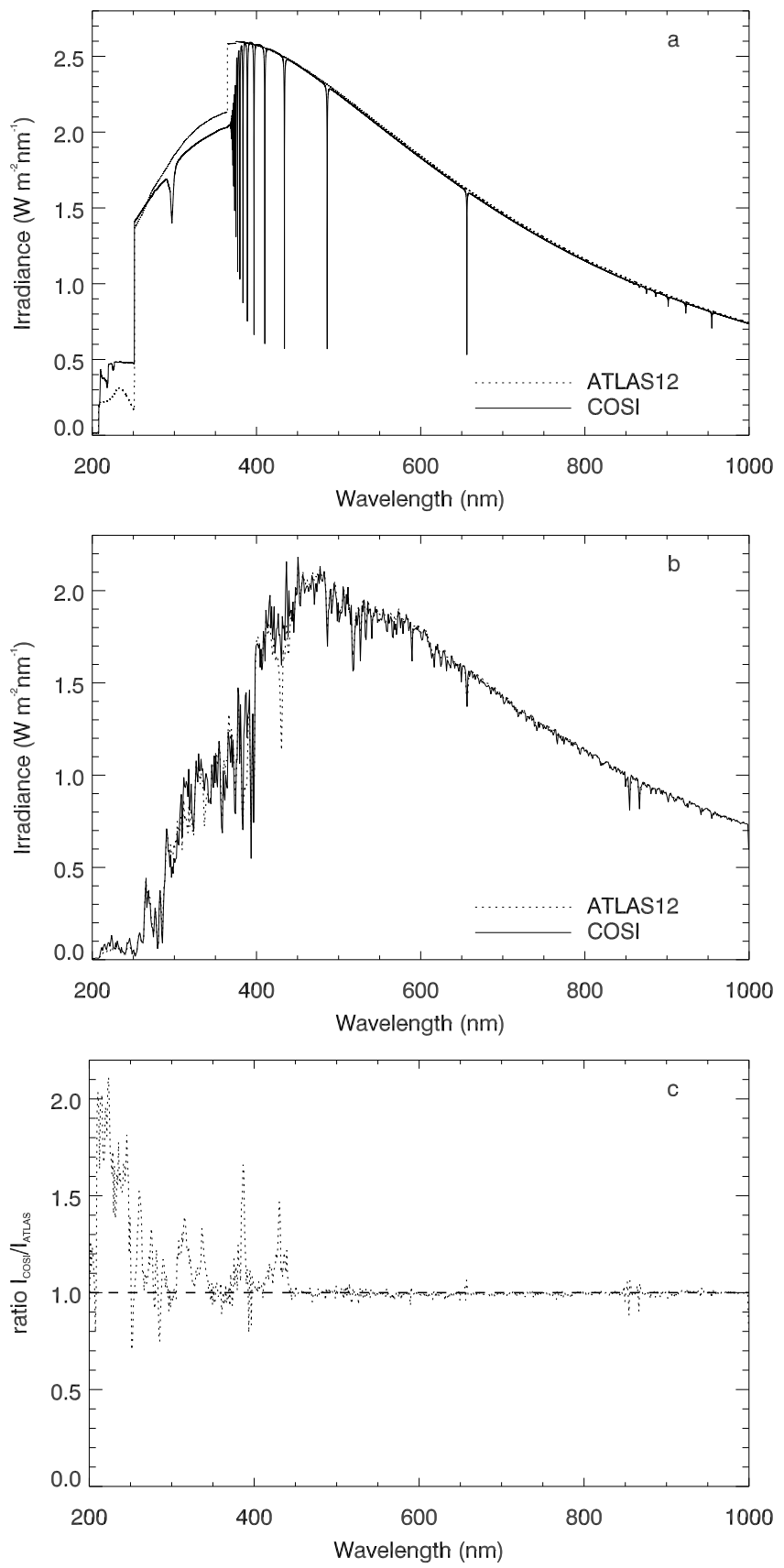

Fig. 3. Panel a) shows the comparison of the continuum calculated with the ATLAS12 code (dotted line) and continuum plus hydrogen lines calculated with COSI in LTE (solid line), based on identical atmosphere structure and abundances. Panel b) gives the corresponding synthetic LTE spectra averaged with a 1-nm boxcar. Panel c) shows the ratio between the COSI and the ATLAS12 calculation, shown in panel b).

(ODFs) of the line opacities. The concept of ODFs was first suggested by Strom \& Kurucz (1966), and for a review see Carbon (1984). To our knowledge, this concept has not yet been used with an iterative scheme in a NLTE calculation.

\subsubsection{Implementation of NLTE-ODFs}

For the calculation of the ODFs the high resolution line opacities need to be calculated over the wavelength range under consideration, which in our case is from 90 to $400 \mathrm{~nm}$. The computing time would be prohibitive to account for each single line in the

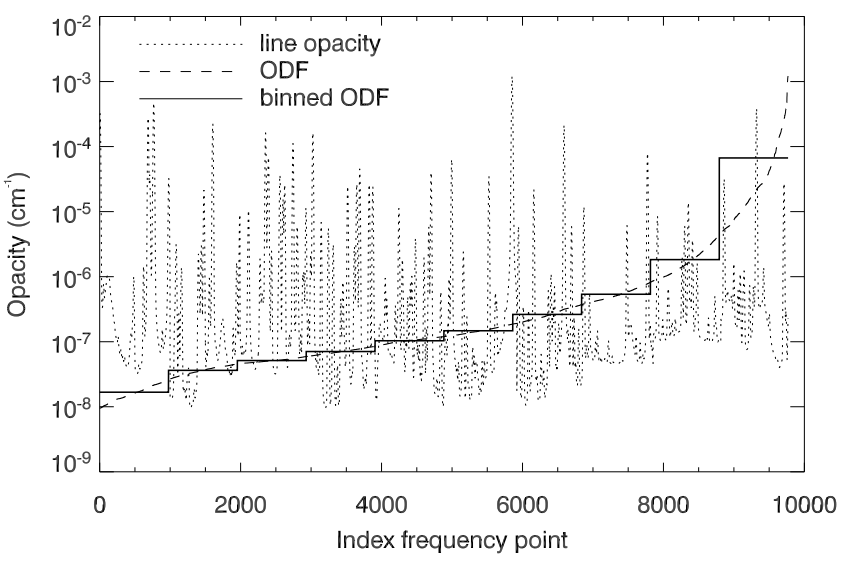

Fig. 4. Line opacities for the wavelength range from 200 to $210 \mathrm{~nm}$ for the depth point where $\tau_{500}=1$. The huge number of frequency points necessary to account for the detailed line opacities is reduced to 10 values by means of the ODFs. This allows the inclusion of the line opacities in the NLTE radiative transfer with a reasonable computational effort.

NLTE solution and therefore we describe all the line opacities by distribution functions over a certain wavelength range $\Delta \lambda$ (in our case $10 \mathrm{~nm}$ ) as a function of line strength. This means that over $\Delta \lambda$ and for each depth point the line opacities are sorted according to their strengths. Then, for a certain number of opacity bins (in our case 10 ) we calculate the mean opacity $\bar{\kappa}_{j}$ for each bin $j$ as given by:

$\bar{\kappa}_{j}=\operatorname{mean}\left(\operatorname{sort}\left(\kappa_{i}\right)\right)_{j}$,

with $i$ being the frequency index of the high resolution opacities. The bins $j$ are then transferred to evenly spaced wavelength points within the wavelength interval under consideration yielding NLTE-ODFs with a $1 \mathrm{~nm}$ resolution. Figure 4 shows the high resolution line opacities (dotted line) from 200 to $210 \mathrm{~nm}$ at the depth where $\tau_{500}=1$. Also shown are the calculated ODF (dashed line) and the binned mean opacity (solid line), again for the same depth point. Thus, we include values of averaged line opacity, representing the high resolution line opacity, in the NLTE calculation.

To avoid the excess of the chromospheric emission for lines that are calculated in LTE, the line emissivity for layers outward the temperature minimum is set to the value calculated from the Planck function at the temperature minimum. We are aware that this approach clearly underestimates the emissivity for many upper chromosphere lines and it will be improved in the future through the inclusion of more levels in the NLTE calculation and/or by an approximate treatment of the NLTE effects, e.g. using the modified excitation temperatures as introduced by Schmutz (1991).

Although the NLTE-ODF distribution function is calculated from LTE level populations there is an indirect effect of the NLTE population through changes of the ionization equilibrium, i.e. all of the level populations that are treated in LTE with respect to the NLTE ground state population (cf. Table 2) are indirectly affected by the NLTE effects. Thus the inclusion of the ODFs changes the line strengths of the emergent spectrum, which in turn leads to a different set of ODFs. Therefore, self-consistent ODFs can only be calculated iteratively. We have investigated to what extent a set of successive iterations of ODFs influences the population values of the explicit NLTE levels. We also studied how many iterations are necessary to find a solution for which the ODFs are practically unchanged in a next iteration. The changes of the population numbers calculated without and 

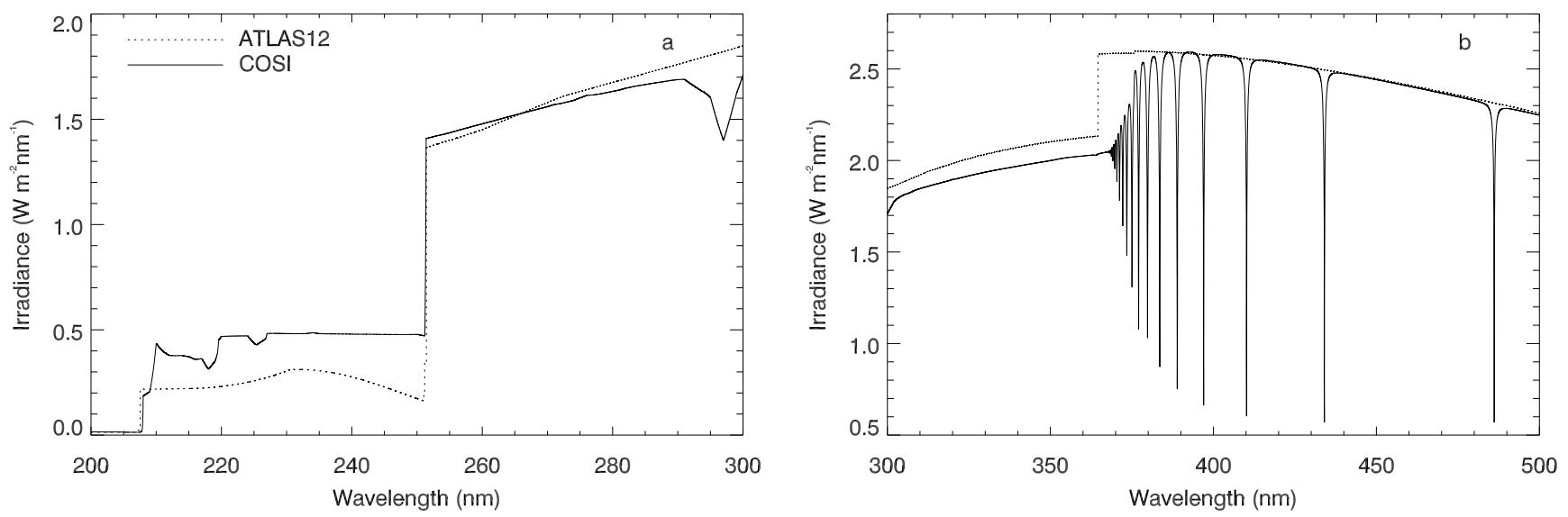

Fig. 5. Same as Fig. 3a for the wavelength range 200 to $300 \mathrm{~nm}$ (panel a)), and 300 to $500 \mathrm{~nm}$ (panel b)). The differences are due to different photoionization cross sections implemented in COSI. The lines in panel b) are the hydrogen lines longward of the Balmer jump.
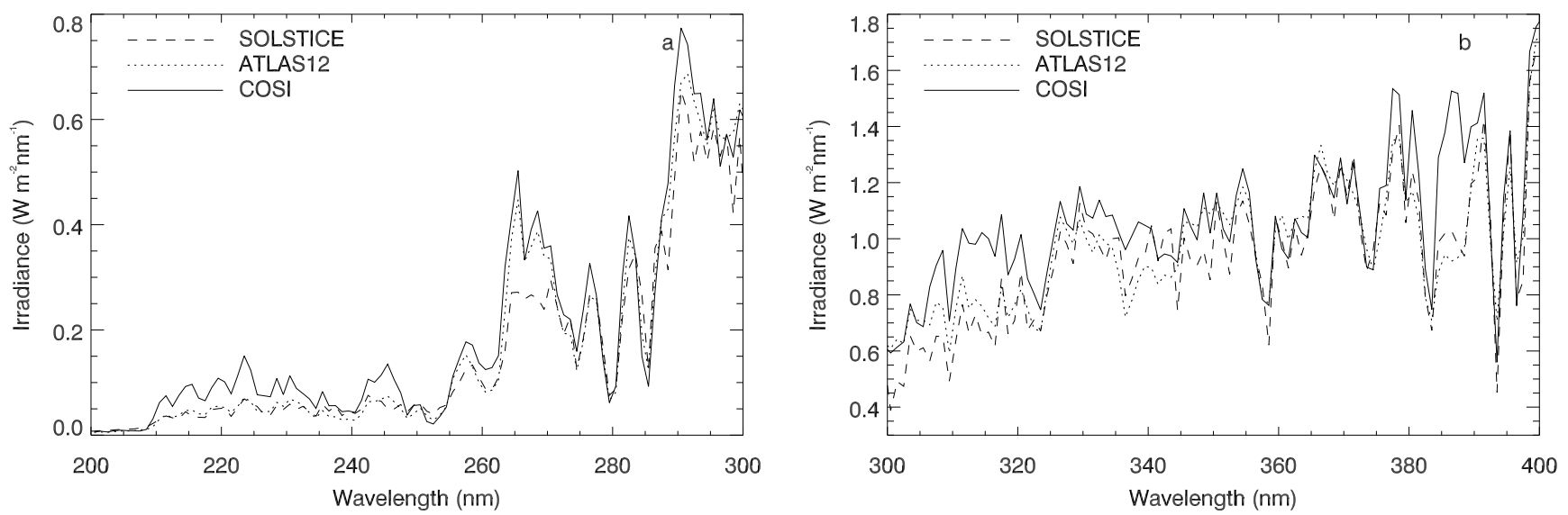

Fig. 6. Same as Fig. 3b plus a comparison with the SOLSTICE measurements (dashed line). The synthetic spectra are convolved with 1-nm boxcar filters to reproduce the resolution of the observation. The higher flux between 210 and $235 \mathrm{~nm}$ can be explained by missing continuum and line opacity. The discrepancy between 385 and $390 \mathrm{~nm}$ is due to the CN-band not calculated with COSI.

with the first set of ODFs are up to a factor 30. The next iteration leads to changes of 1.5 , the second and third iterations show maximal changes of a factor of 1.002. As the population numbers are practically unchanged between the second and third set of ODFs, we conclude that two iterations are sufficient to find a self-consistent distribution of ODFs (Haberreiter 2006).

\subsubsection{Results with NLTE-ODFs}

In this section we present NLTE calculations carried out with COSI, using the F1999 model by Fontenla et al. (1999) along with the F2007 abundance values, and the NLTE-ODFs as described in the section above. Figure 7 shows the effect of the NLTE-ODFs on the emergent continuum spectrum. The dotted line represents the continuum spectrum calculated without NLTE-ODFs, and the solid line shows the spectrum with NLTE-ODFs. The implementation of the NLTE-ODFs leads to a considerable decrease in the flux from $\sim 100$ to $200 \mathrm{~nm}$. This is due to the fact that the NLTE-ODFs increase the opacity. Consequently, the height where $\tau$ equals 1 is higher up in the solar atmosphere where the temperature is lower than in the case for the pure continuum opacity. Thus, the UV flux is decreased and the elements, in particular the metals, are less ionized. The increase in neutral atoms in turn leads to an increase in the continuum opacity and thus to a decrease in the continuum flux. It is important to note that the negative hydrogen opacity strongly depends on the electron density. As the ionization decreases, less negative hydrogen is formed due to a decrease in the electron density in some layers, leading to a slight decrease in the continuum flux in the optical wavelength range (Fig. 7b). Note that COSI calculates the negative hydrogen in full NLTE, i.e. in a simultaneous solution together with all other NLTE levels as given in Table 2.

Figure 8 shows the corresponding line spectrum calculated in NLTE with COSI together with SOLSTICE measurements and ATLAS12 calculation. In order to compensate for the missing opacities, which is also partly present in the COSI LTE calculation, the Doppler broadening was increased from $1.5 \mathrm{~km} \mathrm{~s}^{-1}$ to $15 \mathrm{~km} \mathrm{~s}^{-1}$ in the wavelength range from 170 to $210 \mathrm{~nm}$, from $1.5 \mathrm{~km} \mathrm{~s}^{-1}$ to $8 \mathrm{~km} \mathrm{~s}^{-1}$ from 210 to $250 \mathrm{~nm}$, and from $1.5 \mathrm{~km} \mathrm{~s}^{-1}$ to $3 \mathrm{~km} \mathrm{~s}^{-1}$ from 250 to $400 \mathrm{~nm}$. For the wavelength range from 300 to $400 \mathrm{~nm}$ the COSI calculation is in reasonable agreement with the observation. To reproduce the observation it is clear that the implementation of the NLTE-ODFs is important, because without them the emergent flux would be considerably higher, as already shown in Fig. 7. Nevertheless, the remaining issues about the missing opacities need further investigation. For future calculations the inclusion of molecular lines and additional continuum opacities such as $\mathrm{CH}, \mathrm{OH}$ and $\mathrm{CN}$ photodissociation are necessary. 

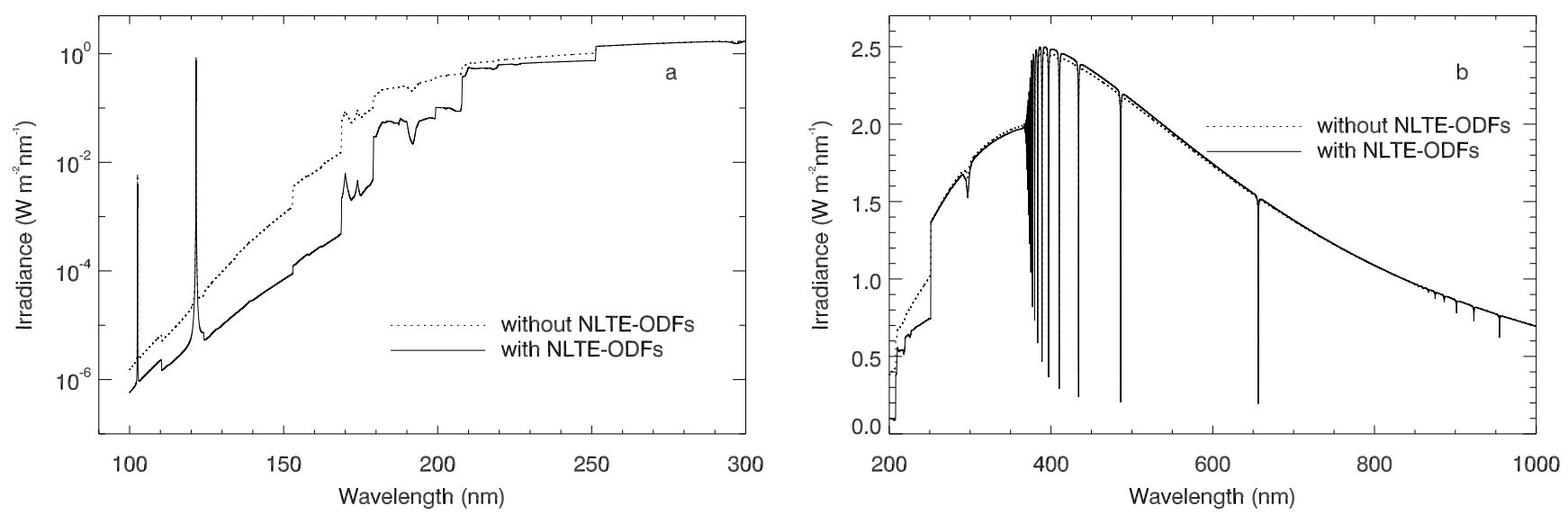

Fig. 7. Comparison of the continuum calculations carried out with COSI in NLTE, using the F1999 model atmosphere structure to analyze the effect of the NLTE-ODFs on the calculated continuum.
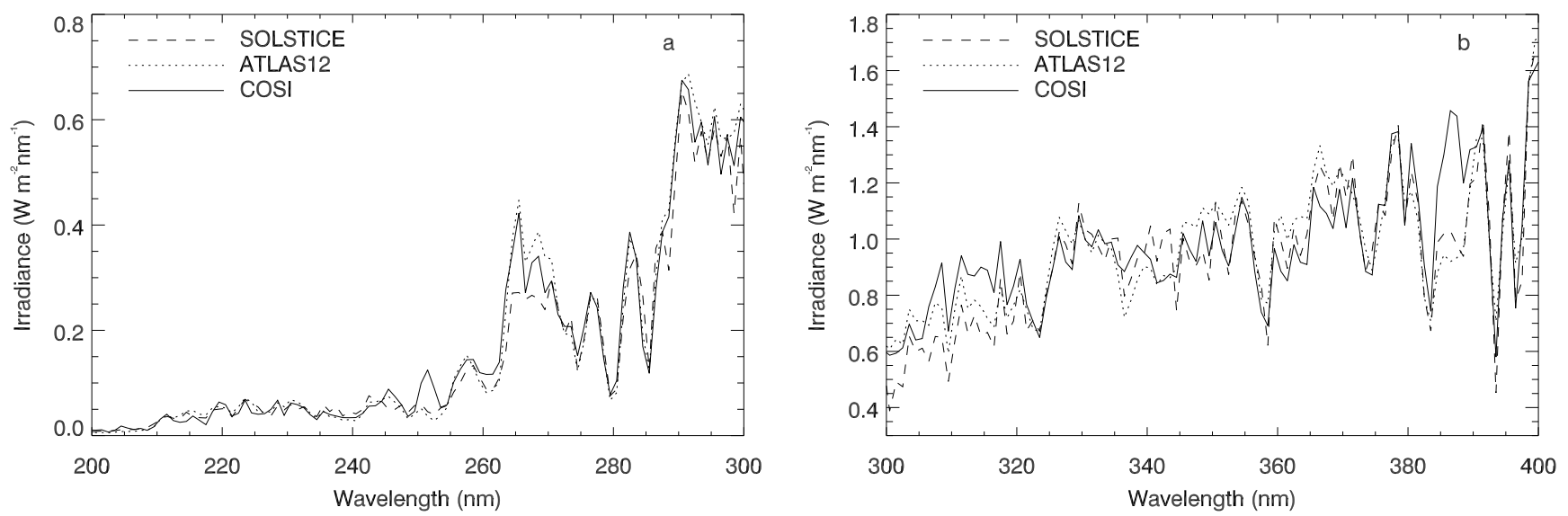

Fig. 8. Comparison of the synthetic spectrum calculated with COSI in NLTE using F1999 as atmosphere structure with the SOLSTICE measurements (dashed line) and the ATLAS12 calculation. The synthetic spectra are convolved with a 1-nm boxcar to reproduce the resolution of the observation.

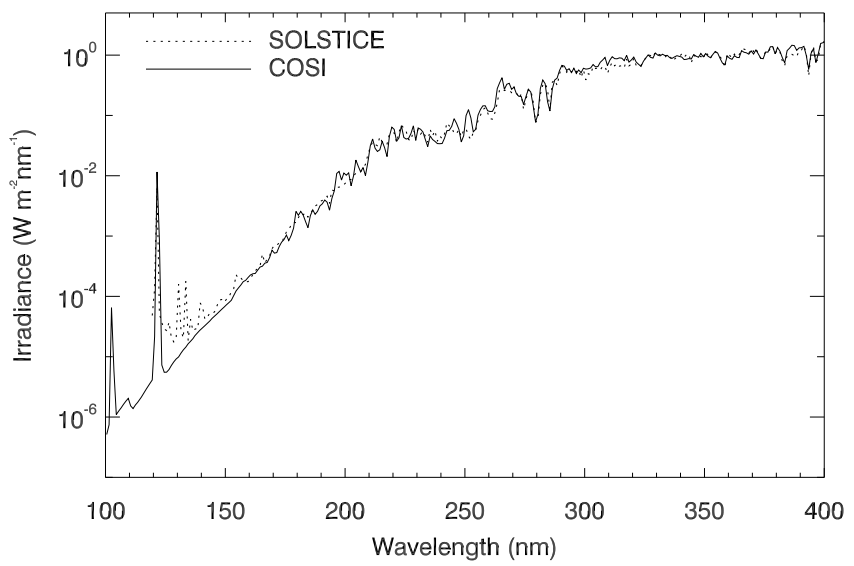

Fig. 9. Comparison of the synthetic spectrum calculated with COSI in NLTE with the F1999 atmosphere structure (solid line) together with the SOLSTICE observation at solar minimum on April 3, 1997 (dashed line).

Finally, Fig. 9 shows the comparison of the line spectrum calculated in NLTE with COSI along with SOLSTICE measurements for the wavelength range from 100 to $400 \mathrm{~nm}$. The overall agreement between the calculation and the observation is good. However, the continuum around the Lyman- $\alpha$ line is considerably lower than the observation. One of the reasons for this is clearly the missing contribution of the quiet Sun network and also enhanced network to the calculated spectrum. Also, the fact that our approach underestimates the emissivity of the chromospheric emission lines, can further explain the discrepancy below $150 \mathrm{~nm}$. Nevertheless, the general agreement above $150 \mathrm{~nm}$, in particular the continuum slope, clearly shows that the inclusion of the line opacities is essential for the calculation of the solar spectrum in the UV.

\section{Discussion}

In the current version of COSI we have calculated the NLTE-ODFs with line opacities in LTE with respect to the ground state of the ion. For all heights above the temperature minimum the emissivity is set to $\epsilon_{L, \lambda}=B_{\lambda, T_{\min }} \kappa_{L, \lambda}$, with $B$ being the Planck function and $L$ the depth point index of the atmosphere structure. In F1999 the layers around $T_{\min }$ are in nearLTE conditions which makes our approach suitable for this and similar stellar atmosphere models. However, if we would calculate an atmosphere with strong departures from LTE, as e.g. SRPM 305, our assumptions would lead to a substantial overestimation of the opacity and at the same time an underestimation of the source function above the temperature minimum, which in turn would lead to a much lower UV continuum intensity than in the observations shown in the calculations by 
Fontenla et al. (2007). In order to address such structures the NLTE effects have to be accounted for in more detail.

Haberreiter et al. (2005) presented a comparison of the calculated Lyman- $\alpha$ line strength as a function of time over two solar cycles with its observed strength as compiled by Woods et al. (2000). Their calculations are based on the same F1999 COSI model as discussed above. They found that the synthetic Lyman- $\alpha$ line is about a factor of 2 too weak. The discrepancy has been found in part to be due to ambipolar diffusion of protons and neutral hydrogen as implemented in F1999 and the later atmosphere structure SRPM305 by Fontenla et al. (2007) but not yet in the COSI code. Another important contribution which leads to an increased Lyman $\alpha$ line strength is the expansion of the area of active regions when a line is formed higher up in the solar atmosphere (Schöll et al. 2008).

\section{Conclusions}

We presented the NLTE radiative transfer code COSI for the calculation of the spectral solar energy distribution and validated its performance in LTE against ATLAS12 calculations and observed solar spectra. The results show that COSI correctly calculates the spectra in LTE. Furthermore, we have introduced the new concept of NLTE-ODFs. This new technique allows all line opacities to be included in the NLTE radiative transfer. The fast convergence of the NLTE-ODFs does not significantly increase the computing time. We summarize that by including NLTEODFs in the radiative transfer code COSI the calculations of the solar spectra are improved substantially. It is important to note that this new concept is not limited to specific atoms, and can in principle also be used in an iterative scheme to derive stellar atmosphere structures.

Acknowledgements. This work was supported through ETH Polyproject Variability of the Sun and Global Climate and SNF Project No. 200020-109420. We would like to give special thanks to Robert Kurucz for providing the synthetic spectra calculated with ATLAS12, as well as the line opacities. We very warmly acknowledge that he kindly answered the many questions we had concerning the solar spectra. We are also grateful to Juan Fontenla for providing the atmosphere structures and many fruitful discussions.

\section{References}

Allende Prieto, C., Hubeny, I., \& Lambert, D. L. 2003a, ApJ, 591, 1192

Allende Prieto, C., Lambert, D. L., Hubeny, I., \& Lanz, T. 2003b, ApJS, 147, 363

Anderson, L. S. 1989, ApJ, 339, 558

Anderson, L. S., \& Athay, R. G. 1989, ApJ, 346, 1010

Asplund, M., Nordlund, A., Trampedach, R., Allende Prieto, C., \& Stein, R. F. 2000, A\&A, 359, 729

Asplund, M., Grevesse, N., Sauval, A. J., Allende Prieto, C., \& Blomme, R. 2005, A\&A, 431, 693

Avrett, E. H., \& Loeser, R. 2008, ApJS, 175, 229

Ayres, T. R., Plymate, C., \& Keller, C. U. 2006, ApJS, 165, 618

Bautista, M. A. 1997, A\&AS, 122, 167

Bautista, M. A., \& Pradhan, A. K. 1997, A\&AS, 126, 365

Berger, J. M. 1956, ApJ, 124, 550

Carbon, D. F. 1984, Line blanketing (Methods in Radiative Transfer), 395

Carlsson, M. 1986, Uppsala Astronomical Observatory Reports, 33

Collet, R., Asplund, M., \& Thevenin, F. 2005, A\&A, 442, 643

de Marco, O., \& Schmutz, W. 1999, A\&A, 345, 163

Dreizler, S., \& Werner, K. 1993, A\&A, 278, 199
Egorova, T., Rozanov, E., Manzini, E., et al. 2004, Geophys. Res. Lett., 31, 6119 Fontenla, J., White, O. R., Fox, P. A., Avrett, E. H., \& Kurucz, R. L. 1999, ApJ, 518,480

Fontenla, J. M., Balasubramaniam, K. S., \& Harder, J. 2007, ApJ, 667, 1243

Fox, P. A., Fontenla, J. M., \& White, O. R. 2004, Adv. Space Res., 34, 231

Gray, D. F. 1992, The Observation and Analysis of Stellar Photospheres (Cambridge, UK: Cambridge University Press)

Haberreiter, M. 2006, Ph.D. Thesis (ETH Zürich, Switzerland: Cuvillier Verlag Gottingen)

Haberreiter, M., Rozanov, E., Rüedi, I., \& Schmutz, W. 2003, in Stellar Atmosphere Modeling, ASP Conf. Ser., 288, 165

Haberreiter, M., Krivova, N. A., Schmutz, W., \& Wenzler, T. 2005, Adv. Space Res., 35, 365

Haberreiter, M., Kosovichev, A. G., \& Schmutz, W. 2008, ApJ, 675, L53

Hamann, W.-R., \& Schmutz, W. 1987, A\&A, 174, 173

Hubeny, I. 1981, A\&A, 98, 96

Hubeny, I. 1988, Comp. Phys. Comm., 52, 103

Hubeny, I., \& Lanz, T. 1995, ApJ, 439, 875

Hubeny, I., \& Lites, B. W. 1995, ApJ, 455, 376

Irwin, A. W. 1981, ApJS, 45, 621

Jefferies, J. T. 1968, Spectral line formation, A Blaisdell Book in the Pure and Applied Sciences (Waltham, Mass.: Blaisdell)

John, T. L. 1988, A\&A, 193, 189

Karzas, W. J., \& Latter, R. 1961, ApJS, 6, 167

Kilifarska, N. A., \& Haigh, J. D. 2005, J. Atm. Terrestrial Phys., 67, 241

Koester, D., Vauclair, G., Dolez, N., et al. 1985, A\&A, 149, 423

Koesterke, L., Allende Prieto, C., \& Lambert, D. L. 2008, ApJ, 680, 764

Krivova, N. A., \& Solanki, S. K. 2005, Adv. Space Res., 35, 361

Kurucz, R. L. 1991, in Stellar Atmospheres - Beyond Classical Models, NATO ASIC Proc., 341, 441

Kurucz, R. L. 2005, Mem. Soc. Astron. Ital. Supp., 8, 189

Kurucz, R. L. 2006, personal communication

Labitzke, K. 2005, J. Atm. Terrestrial Phys., 67, 45

Lambert, D. L., \& Pagel, B. E. J. 1968, MNRAS, 141, 299

Lockwood, M., \& Fröhlich, C. 2007, Proc. R. Soc. A, 463, 2447

Mihalas, D. 1967, ApJ, 149, 169

Mihalas, D. 1978, Stellar atmospheres (San Francisco: W. H. Freeman and Co.)

Peraiah, A. 2001, An Introduction to Radiative Transfer, by Annamaneni Peraiah (Cambridge, UK: Cambridge University Press), 492

Rottman, G. J., Woods, T. N., \& Sparn, T. P. 1993, J. Geophys. Res., 98, 10667

Rozanov, E., Egorova, T., Fröhlich, C., et al. 2002, in Proceedings of the SOHO 11 Symposium on From Solar Min to Max: Half a Solar Cycle with SOHO, ed. A. Wilson, ESA SP-508 (Noordwijk: ESA Publications Division), 181

Rozanov, E., Egorova, T., Schmutz, W., \& Peter, T. 2006, J. Atm. Terrestrial Phys., 68, 2203

Schaerer, D., \& Schmutz, W. 1994, A\&A, 288, 231

Schmutz, W. 1991, in Stellar Atmospheres - Beyond Classical Models, NATO ASIC Proc., 341, 191

Schmutz, W. 1997, A\&A, 321, 268

Schmutz, W., Hamann, W.-R., \& Wessolowski, U. 1989, A\&A, 210, 236

Schmutz, W., Leitherer, C., Hubeny, I., et al. 1991, ApJ, 372, 664

Schöll, et al. 2008, in preparation

Seaton, M. J. 1960, Rep. Progr. Phys., 23, 313

Seaton, M. J., Yan, Y., Mihalas, D., \& Pradhan, A. K. 1994, MNRAS, 266, 805

Shindell, D. T., Schmidt, G. A., Mann, M. E., Rind, D., \& Waple, A. 2001, Science, 294, 2149

Shindell, D. T., Schmidt, G. A., Miller, R. L., \& Mann, M. E. 2003, J. Climate, 16,4094

Short, C. I., \& Hauschildt, P. H. 2005, ApJ, 618, 926

Strom, S. E., \& Kurucz, R. 1966, AJ, 71, 181

Traving, G., Baschek, B., \& Holweger, H. 1966, Astronomische Abhandlungen der Hamburger Sternwarte, 8,1

Uitenbroek, H. 2001, ApJ, 557, 389

Uitenbroek, H. 2002, ApJ, 565, 1312

Vernazza, J. E., Avrett, E. H., \& Loeser, R. 1981, ApJS, 45, 635

Wenzler, T., Solanki, S. K., \& Krivova, N. A. 2005, A\&A, 432, 1057

Wenzler, T., Solanki, S. K., Krivova, N. A., \& Fröhlich, C. 2006, A\&A, 460, 583

Woods, T. N., Rottman, G. J., \& Ucker, G. J. 1993, J. Geophys. Res., 98, 10679

Woods, T. N., Tobiska, W. K., Rottman, G. J., \& Worden, J. R. 2000, J. Geophys. Res., 105, 27195 
M. Haberreiter et al.: NLTE calculations of the solar atmosphere, Online Material $p 1$

Table 2. Levels of the atomic model explicitly treated in NLTE by COSI. The columns denote the ion, level, atomic number AN, electron configuration and spectroscopic term designation, the ionization wavelength $\lambda_{\text {ion }}$ calculated from the measured energies from the NIST database, where * detotes ionization to an excited state of the next higher ion, the ionization potential $E_{\text {ion }}$ of the ground state, the weighted mean of the level energy $E$, the statistical weight $g$ of the model configuration, and the source of the photoionization cross sections.

\begin{tabular}{|c|c|c|c|c|c|c|c|c|c|}
\hline Ion & Level & $\mathrm{AN}$ & Configuration & Term & $\lambda_{\text {ion }}(\AA)$ & $E_{\text {ion }}\left(\mathrm{cm}^{-1}\right)$ & $E\left(\mathrm{~cm}^{-1}\right)$ & $g$ & Source \\
\hline $\mathrm{H}^{-}$ & 1 & 1 & $1 \mathrm{~s}$ & ${ }^{1} \mathrm{~S}$ & 16414.54 & 6090.50 & 0.0 & 2 & $\mathrm{~J} 88^{1}$ \\
\hline $\mathrm{HI}_{\mathrm{I}}$ & 1 & 1 & $1 \mathrm{~s}$ & ${ }^{2} \mathrm{~S}$ & 911.77 & 109677.38 & 0.0 & 2 & $\mathrm{MIH}^{2}$ \\
\hline $\mathrm{HI}$ & 2 & 1 & $2 p$ & ${ }^{2} \mathrm{P}^{\circ}$ & 3645.49 & & 82258.04 & 8 & MIH \\
\hline $\mathrm{HI}$ & 3 & 1 & $2 \mathrm{~s}$ & ${ }^{2} \mathrm{~S}$ & 8200.65 & & 97491.00 & 18 & MIH \\
\hline H I & 4 & 1 & $3 p$ & ${ }^{2} \mathrm{P}^{\circ}$ & 14576.68 & & 102822.54 & 32 & MIH \\
\hline H I & 5 & 1 & $3 \mathrm{~s}$ & ${ }^{2} \mathrm{~S}$ & 22773.63 & & 105290.28 & 50 & MIH \\
\hline H I & 6 & 1 & $3 d$ & ${ }^{2} \mathrm{D}$ & 32791.60 & & 106630.79 & 72 & MIH \\
\hline H I & 7 & 1 & $4 p$ & ${ }^{2} \mathrm{P}^{\circ}$ & 44630.70 & & 107439.07 & 98 & MIH \\
\hline $\mathrm{HI}$ & 8 & 1 & $4 \mathrm{~s}$ & ${ }^{2} \mathrm{~S}$ & 58291.00 & & 107963.67 & 128 & MIH \\
\hline H I & 9 & 1 & $4 d$ & ${ }^{2} \mathrm{D}$ & 73772.54 & & 108323.34 & 162 & $\mathrm{MIH}$ \\
\hline $\mathrm{HI}$ & 10 & 1 & $4 \mathrm{f}$ & ${ }^{2} \mathrm{P}^{\circ}$ & 91075.35 & & 108580.61 & 200 & MIH \\
\hline H II & 1 & 1 & - & - & & & 0.0 & 1 & - \\
\hline He I & 1 & 2 & $1 \mathrm{~s}^{2}$ & ${ }^{1} \mathrm{~S}$ & 504.25 & 8200.65 & 0.0 & 1 & $\mathrm{KOE}^{3}$ \\
\hline He I & 2 & 2 & $1 \mathrm{~s} 2 \mathrm{~s}$ & ${ }^{3} \mathrm{~S}$ & 2601.00 & & 159856.07 & 3 & KOE \\
\hline He I & 3 & 2 & $1 \mathrm{~s} 2 \mathrm{~s}$ & ${ }^{1} \mathrm{~S}$ & 3122.27 & & 166277.55 & 1 & $\mathrm{KOE}$ \\
\hline $\mathrm{He} \mathrm{I}$ & 4 & 2 & $1 s 2 p$ & ${ }^{3} \mathrm{P}^{\circ}$ & 3422.37 & & 169086.94 & 7 & $\mathrm{KOE}$ \\
\hline He I & 5 & 2 & $1 \mathrm{~s} 2 \mathrm{p}$ & ${ }^{1} \mathrm{P}^{\circ}$ & 3680.26 & & 171135.00 & 3 & $\mathrm{KOE}$ \\
\hline He I & 6 & 2 & $1 \mathrm{~s} 3 \mathrm{~s}$ & ${ }^{3} \mathrm{~S}$ & 6634.61 & & 183236.89 & 3 & $\mathrm{KOE}$ \\
\hline He I & 7 & 2 & $1 \mathrm{~s} 3 \mathrm{~s}$ & ${ }^{1} \mathrm{~S}$ & 7437.69 & & 184864.55 & 1 & $\mathrm{KOE}$ \\
\hline He I & 8 & 2 & $1 s 3 p$ & ${ }^{3} \mathrm{P}^{\circ}$ & 7846.22 & & 185564.68 & 7 & KOE \\
\hline He I & 9 & 2 & $1 \mathrm{~s} 3 \mathrm{~d}$ & ${ }^{3} \mathrm{D}$ & 8191.29 & & 186101.65 & 13 & $\mathrm{KOE}$ \\
\hline He I & 10 & 2 & $1 \mathrm{~s} 3 \mathrm{~d}$ & ${ }^{1} \mathrm{D}$ & 8193.59 & & 186105.07 & 5 & $\mathrm{KOE}$ \\
\hline He II & 1 & 2 & $1 \mathrm{~s}$ & ${ }^{2} \mathrm{~S}$ & & 438908.85 & 0.0 & 2 & - \\
\hline Li I & 1 & 3 & {$[\mathrm{He}] 2 \mathrm{~s}$} & ${ }^{2} \mathrm{~S}$ & 2298.90 & 43487.15 & 0.0 & 2 & $\mathrm{Hyd}^{4}$ \\
\hline Li II & 1 & 3 & {$[\mathrm{He}]$} & ${ }^{1} \mathrm{~S}$ & & & 0.0 & 1 & - \\
\hline Be I & 1 & 4 & {$[\mathrm{He}] 2 \mathrm{~s}^{2}$} & ${ }^{1} \mathrm{~S}$ & 1329.92 & 75192.64 & 0.0 & 1 & Hyd \\
\hline Be II & 1 & 4 & {$[\mathrm{He}] 2 \mathrm{~s}$} & ${ }^{2} \mathrm{~S}$ & & & 0.0 & 2 & - \\
\hline $\mathrm{B}_{\mathrm{I}}$ & 1 & 5 & {$[\mathrm{He}] 2 \mathrm{~s}^{2} 2 \mathrm{p}$} & ${ }^{2} \mathrm{P}^{\circ}$ & 1494.14 & 66928.10 & 0.0 & 2 & Hyd \\
\hline B II & 1 & 5 & {$[\mathrm{He}] 2 \mathrm{~s}^{2}$} & ${ }^{1} \mathrm{~S}$ & & & 0.0 & 1 & - \\
\hline $\mathrm{CI}$ & 1 & 6 & {$[\mathrm{He}] 2 \mathrm{~s}^{2} 2 \mathrm{p}^{2}$} & ${ }^{3} \mathrm{P}$ & 1101.79 & 90820.42 & 29.57 & 9 & $\mathrm{OP}^{5}$ \\
\hline $\mathrm{CI}_{\mathrm{I}}$ & 2 & 6 & {$[\mathrm{He}] 2 \mathrm{~s}^{2} 2 \mathrm{p}^{2}$} & ${ }^{1} \mathrm{D}$ & 1240.72 & & 10192.63 & 5 & $\mathrm{OP}$ \\
\hline $\mathrm{CI}$ & 3 & 6 & {$[\mathrm{He}] 2 \mathrm{~s}^{2} 2 \mathrm{p}^{2}$} & ${ }^{1} \mathrm{~S}$ & 1446.28 & & 21648.01 & 1 & $\mathrm{OP}$ \\
\hline $\mathrm{C}_{\text {II }}$ & 1 & 6 & {$[\mathrm{He}] 2 \mathrm{~s}^{2} 2 \mathrm{p}$} & ${ }^{2} \mathrm{P}^{\circ}$ & & & 0.00 & 6 & - \\
\hline N I & 1 & 7 & {$[\mathrm{He}] 2 \mathrm{~s}^{2} 2 \mathrm{p}^{3}$} & ${ }^{4} S^{\circ}$ & 852.62 & 117314.58 & 0.0 & 4 & Hyd \\
\hline N II & 1 & 7 & {$[\mathrm{He}] 2 \mathrm{~s}^{2} 2 \mathrm{p}^{2}$} & ${ }^{3} \mathrm{P}$ & & & 0.0 & 9 & - \\
\hline OI & 1 & 8 & {$[\mathrm{He}] 2 \mathrm{~s}^{2} 2 \mathrm{p}^{4}$} & ${ }^{3} \mathrm{P}$ & 911.73 & 109837.02 & 77.97 & 9 & Hyd \\
\hline O II & 1 & 8 & {$[\mathrm{He}] 2 \mathrm{~s}^{2} 2 \mathrm{p}^{3}$} & ${ }^{4} S^{\circ}$ & & & 0.0 & 4 & - \\
\hline FI & 1 & 9 & {$[\mathrm{He}] 2 \mathrm{~s}^{2} 2 \mathrm{p}^{5}$} & ${ }^{3} \mathrm{P}^{\circ}$ & 712.99 & 140524.5 & 134.7 & 6 & Hyd \\
\hline F II & 1 & 9 & {$[\mathrm{He}] 2 \mathrm{~s}^{2} 2 \mathrm{p}^{4}$} & ${ }^{3} \mathrm{P}$ & & & 0.0 & 9 & - \\
\hline $\mathrm{Ne} I$ & 1 & 10 & {$[\mathrm{He}] 2 \mathrm{~s}^{2} 2 \mathrm{p}^{6}$} & ${ }^{1} \mathrm{~S}$ & 574.94 & 173929.75 & & 1 & Hyd \\
\hline $\mathrm{Ne}$ II & 1 & 10 & {$[\mathrm{He}] 2 \mathrm{~s}^{2} 2 \mathrm{p}^{5}$} & ${ }^{2} \mathrm{P}^{\circ}$ & & & 0.0 & 6 & - \\
\hline $\mathrm{NaI}$ & 1 & 11 & {$[\mathrm{Ne}] 3 \mathrm{~s}$} & ${ }^{2} \mathrm{~S}$ & 2411.91 & 41449.62 & 0.00 & 2 & $\mathrm{OP}$ \\
\hline $\mathrm{Na} I$ & 2 & 11 & {$[\mathrm{Ne}] 3 \mathrm{p}$} & ${ }^{2} \mathrm{P}^{\circ}$ & 4083.51 & & 16967.64 & 6 & $\mathrm{OP}$ \\
\hline $\mathrm{Na} I$ & 3 & 11 & {$[\mathrm{Ne}] 4 \mathrm{~s}$} & ${ }^{2} \mathrm{~S}$ & 6363.72 & & 25739.99 & 2 & $\mathrm{OP}$ \\
\hline $\mathrm{Na} I$ & 4 & 11 & {$[\mathrm{Ne}] 3 \mathrm{~d}$} & ${ }^{2} \mathrm{D}$ & 8143.14 & & 29172.86 & 10 & $\mathrm{OP}$ \\
\hline $\mathrm{Na} I$ & 5 & 11 & {$[\mathrm{Ne}] 4 \mathrm{p}$} & ${ }^{2} \mathrm{P}^{\circ}$ & 8942.85 & & 30270.72 & 6 & $\mathrm{OP}$ \\
\hline $\mathrm{Na} I$ & 6 & 11 & {$[\mathrm{Ne}] 5 \mathrm{~s}$} & ${ }^{2} S$ & 12119.24 & & 33200.68 & 2 & $\mathrm{OP}$ \\
\hline $\mathrm{Na} I$ & 7 & 11 & {$[\mathrm{Ne}] 4 \mathrm{~d}$} & ${ }^{2} \mathrm{D}$ & 14486.66 & & 34548.74 & 10 & $\mathrm{OP}$ \\
\hline $\mathrm{Na} I$ & 8 & 11 & {$[\mathrm{Ne}] 4 \mathrm{f}$} & ${ }^{2} \mathrm{~F}^{\circ}$ & 14567.25 & & 34586.92 & 14 & $\mathrm{OP}$ \\
\hline $\mathrm{NaI}$ & 9 & 11 & {$[\mathrm{Ne}] 5 \mathrm{p}$} & ${ }^{2} \mathrm{P}^{\circ}$ & 15601.91 & & 35042.03 & 6 & $\mathrm{OP}$ \\
\hline $\mathrm{NaI}$ & 10 & 11 & {$[\mathrm{Ne}] 6 \mathrm{~s}$} & ${ }^{2} S$ & 19690.85 & & 36372.62 & 2 & $\mathrm{OP}$ \\
\hline Na II & 1 & 11 & {$[\mathrm{Ne}]$} & ${ }^{1} \mathrm{~S}$ & & & 0.00 & 1 & - \\
\hline Mg I & 1 & 12 & {$[\mathrm{Ne}] 3 \mathrm{~s}^{2}$} & ${ }^{1} \mathrm{~S}$ & 1621.51 & 61671.02 & 0.00 & 1 & $\mathrm{OP}$ \\
\hline $\mathrm{Mg}_{\mathrm{I}}$ & 2 & 12 & {$[\mathrm{Ne}] 3 \mathrm{~s} 3 \mathrm{p}$} & ${ }^{3} \mathrm{P}^{\circ}$ & 2512.26 & & 21877.30 & 9 & $\mathrm{OP}$ \\
\hline $\mathrm{Mg}$ I & 3 & 12 & {$[\mathrm{Ne}] 3 \mathrm{~s} 3 \mathrm{p}$} & ${ }^{1} \mathrm{P}^{\circ}$ & 3755.50 & & 35051.26 & 3 & $\mathrm{OP}$ \\
\hline Mg II & 1 & 12 & {$[\mathrm{Ne}] 3 \mathrm{~s}$} & ${ }^{2} S$ & & & 0.00 & 2 & - \\
\hline $\mathrm{Al} \mathrm{I}$ & 1 & 13 & {$[\mathrm{Ne}] 3 \mathrm{~s}^{2} 3 p$} & ${ }^{2} \mathrm{P}^{\circ}$ & 2077.19 & 48278.20 & 74.71 & 6 & OP \\
\hline $\mathrm{Al}$ II & 1 & 13 & {$[\mathrm{Ne}] 3 \mathrm{~s}^{2}$} & ${ }^{1} \mathrm{~S}$ & & & 0.00 & 1 & - \\
\hline Si I & 1 & 14 & {$[\mathrm{Ne}] 3 \mathrm{~s}^{2} 3 \mathrm{p}^{2}$} & ${ }^{3} \mathrm{P}$ & 1527.92 & 65747.76 & 149.68 & 9 & OP \\
\hline Si I & 2 & 14 & {$[\mathrm{Ne}] 3 \mathrm{~s}^{2} 3 \mathrm{p}^{2}$} & ${ }^{1} \mathrm{D}$ & 1686.36 & & 6298.85 & 5 & $\mathrm{OP}$ \\
\hline Si I & 3 & 14 & {$[\mathrm{Ne}] 3 \mathrm{~s}^{2} 3 \mathrm{p}^{2}$} & ${ }^{1} \mathrm{~S}$ & 1991.88 & & 15394.37 & 1 & $\mathrm{OP}$ \\
\hline
\end{tabular}


M. Haberreiter et al.: NLTE calculations of the solar atmosphere, Online Material $p 2$

Table 2. continued.

\begin{tabular}{|c|c|c|c|c|c|c|c|c|c|}
\hline Ion & Level & $\mathrm{AN}$ & Configuration & Term & $\lambda_{\text {ion }}(\AA)$ & $E_{\text {ion }}\left(\mathrm{cm}^{-1}\right)$ & $E\left(\mathrm{~cm}^{-1}\right)$ & $g$ & Source \\
\hline Si I & 4 & 14 & {$[\mathrm{Ne}] 3 \mathrm{~s} 3 \mathrm{p}^{3}$} & ${ }^{5} \mathrm{~S}^{\circ}$ & $* 1328.47$ & & 33326.04 & 5 & OP \\
\hline $\mathrm{Si}$ I & 5 & 14 & {$[\mathrm{Ne}] 3 \mathrm{~s}^{2} 3 \mathrm{p} 4 \mathrm{~s}$} & ${ }^{3} \mathrm{P}^{\circ}$ & 3875.02 & & 39799.50 & 9 & OP \\
\hline Si I & 6 & 14 & {$[\mathrm{Ne}] 3 \mathrm{~s}^{2} 3 \mathrm{p} 4 \mathrm{~s}$} & ${ }^{1} \mathrm{P}^{\circ}$ & 4062.78 & & 40991.88 & 3 & OP \\
\hline Si II & 1 & 14 & {$[\mathrm{Ne}] 3 \mathrm{~s}^{2}\left({ }^{1} \mathrm{~S}\right) 3 \mathrm{p}$} & ${ }^{2} \mathrm{P}^{\circ}$ & & & 0.00 & 6 & - \\
\hline S I & 1 & 16 & {$[\mathrm{Ne}] 3 \mathrm{~s}^{2} 3 \mathrm{p}^{4}$} & ${ }^{3} \mathrm{P}$ & 1199.85 & 83559.10 & 107.74 & 9 & OP \\
\hline S I & 2 & 16 & {$[\mathrm{Ne}] 3 \mathrm{~s}^{2} 3 \mathrm{p}^{4}$} & ${ }^{1} \mathrm{D}$ & 1347.48 & & 9238.61 & 5 & $\mathrm{OP}$ \\
\hline S I & 3 & 16 & {$[\mathrm{Ne}] 3 \mathrm{~s}^{2} 3 \mathrm{p}^{4}$} & ${ }^{1} \mathrm{~S}$ & 1632.08 & & 22179.95 & 1 & OP \\
\hline S II & 1 & 16 & {$[\mathrm{Ne}] 3 \mathrm{~s}^{2} 3 \mathrm{p}^{3}$} & ${ }^{4} S^{\circ}$ & & & 0.00 & 4 & - \\
\hline PI & 1 & 15 & {$[\mathrm{Ne}] 3 \mathrm{~s}^{2} 3 \mathrm{p}^{3}$} & ${ }^{4} S^{\circ}$ & 1182.30 & 84580.83 & 0.0 & 4 & Hyd \\
\hline$P_{I I}$ & 1 & 15 & {$[\mathrm{Ne}] 3 \mathrm{~s}^{2} 3 \mathrm{p}^{2}$} & ${ }^{3} \mathrm{P}$ & & & 0.0 & 9 & - \\
\hline $\mathrm{Cl}$ I & 1 & 17 & {$[\mathrm{Ne}] 3 \mathrm{~s}^{2} 3 \mathrm{p}^{5}$} & ${ }^{2} \mathrm{P}^{\circ}$ & 961.51 & 104591.0 & 0.0 & 6 & Hyd \\
\hline $\mathrm{Cl}$ II & 1 & 17 & {$[\mathrm{Ne}] 3 \mathrm{~s}^{2} 3 \mathrm{p}^{4}$} & ${ }^{3} \mathrm{P}$ & & & 0.0 & 9 & - \\
\hline Ar I & 1 & 18 & {$[\mathrm{Ne}] 3 \mathrm{~s}^{2} 3 \mathrm{p}^{6}$} & ${ }^{1} \mathrm{~S}$ & 786.72 & 127109.80 & 0.0 & 1 & Hyd \\
\hline Ar II & 1 & 18 & {$[\mathrm{Ne}] 3 \mathrm{~s}^{2} 3 \mathrm{p}^{5}$} & ${ }^{2} \mathrm{P}^{\circ}$ & & & 0.0 & 6 & - \\
\hline K I & 1 & 19 & {$[\mathrm{Ar}] 4 \mathrm{~s}$} & ${ }^{2} \mathrm{~S}$ & 2855.56 & 35009.81 & 0.00 & 2 & Hyd \\
\hline K I & 2 & 19 & {$[\mathrm{Ar}] 4 \mathrm{p}$} & ${ }^{2} \mathrm{P}^{\circ}$ & 4547.06 & & 13023.64 & 6 & Hyd \\
\hline K I & 3 & 19 & {$[\mathrm{Ar}] 5 \mathrm{~s}$} & ${ }^{2} \mathrm{~S}$ & 7149.41 & & 21026.55 & 2 & Hyd \\
\hline K I & 4 & 19 & {$[\mathrm{Ar}] 3 \mathrm{~d}$} & ${ }^{2} \mathrm{D}$ & 7419.51 & & 21535.60 & 10 & Hyd \\
\hline K I & 5 & 19 & {$[\mathrm{Ar}] 5 \mathrm{p}$} & ${ }^{2} \mathrm{P}^{\circ}$ & 9709.85 & & 24713.90 & 6 & Hyd \\
\hline K I & 6 & 19 & {$[\mathrm{Ar}] 4 \mathrm{~d}$} & ${ }^{2} \mathrm{D}$ & 13132.87 & & 27397.50 & 10 & Hyd \\
\hline $\mathrm{K}_{\mathrm{I}}$ & 7 & 19 & {$[\mathrm{Ar}] 6 \mathrm{~s}$} & ${ }^{2} \mathrm{~S}$ & 13225.32 & & 27450.71 & 2 & Hyd \\
\hline K I & 8 & 19 & {$[\mathrm{Ar}] 4 \mathrm{f}$} & ${ }^{2} \mathrm{~F}^{\circ}$ & 14526.59 & & 28127.85 & 14 & Hyd \\
\hline K I & 9 & 19 & {$[\mathrm{Ar}] 6 \mathrm{p}$} & ${ }^{2} \mathrm{P}^{\circ}$ & 16648.27 & & 29004.90 & 6 & Hyd \\
\hline $\mathrm{K}_{\mathrm{I}}$ & 10 & 19 & {$[\mathrm{Ar}] 5 \mathrm{~d}$} & ${ }^{2} \mathrm{D}$ & 20721.96 & & 30185.40 & 10 & Hyd \\
\hline K II & 1 & 19 & {$[\mathrm{Ar}]$} & ${ }^{1} \mathrm{~S}$ & & & 0.00 & 1 & - \\
\hline $\mathrm{CaI}$ & 1 & 20 & {$[\mathrm{Ar}] 4 \mathrm{~s}^{2}$} & ${ }^{1} \mathrm{~S}$ & 2027.60 & 49305.95 & 0.00 & 1 & $\mathrm{OP}$ \\
\hline $\mathrm{Ca} \mathrm{I}$ & 2 & 20 & {$[\mathrm{Ar}] 4 \mathrm{~s} 4 \mathrm{p}$} & ${ }^{3} \mathrm{P}^{\circ}$ & 2936.66 & & 15263.09 & 9 & OP \\
\hline $\mathrm{Ca} \mathrm{I}$ & 3 & 20 & {$[\mathrm{Ar}] 3 \mathrm{~d} 4 \mathrm{~s}$} & ${ }^{1} \mathrm{D}^{\circ}$ & 3641.13 & & 21849.63 & 5 & OP \\
\hline Ca II & 1 & 20 & {$[\mathrm{Ar}] 4 \mathrm{~s}$} & ${ }^{2} S$ & & & 0.00 & 2 & - \\
\hline Sc I & 1 & 21 & {$[\mathrm{Ar}] 3 \mathrm{~d} 4 \mathrm{~s}^{2}$} & ${ }^{2} \mathrm{D}$ & 1896.81 & 52922.0 & 0.0 & 10 & Hyd \\
\hline Sc II & 1 & 21 & {$[\mathrm{Ar}] 3 \mathrm{~d} 4 \mathrm{~s}$} & ${ }^{3} \mathrm{D}$ & & & 0.0 & 15 & - \\
\hline Ti I & 1 & 22 & {$[\mathrm{Ar}] 3 \mathrm{~d}^{2} 4 \mathrm{~s}^{2}$} & ${ }^{3} \mathrm{~F}$ & 1831.05 & 55010.0 & 0.0 & 21 & Hyd \\
\hline Ti II & 1 & 22 & {$[\mathrm{Ar}] 3 \mathrm{~d}^{2} 4 \mathrm{~s}$} & ${ }^{4} \mathrm{~F}$ & & & 0.0 & 28 & - \\
\hline$V_{\text {I }}$ & 1 & 23 & {$[\mathrm{Ar}] 3 \mathrm{~d}^{3} 4 \mathrm{~s}^{2}$} & ${ }^{4} \mathrm{~F}$ & 1861.26 & 54360.0 & 316.484 & 30 & Hyd \\
\hline V II & 1 & 23 & {$[\mathrm{Ar}] 3 \mathrm{~d}^{4}$} & ${ }^{5} \mathrm{D}$ & & & 0.0 & 25 & - \\
\hline $\mathrm{Cr} I$ & 1 & 24 & {$[\mathrm{Ar}] 3 \mathrm{~d}^{5} 4 \mathrm{~s}$} & ${ }^{7} \mathrm{~S}$ & 1832.32 & 54575.6 & 0.0 & 7 & Hyd \\
\hline Cr II & 1 & 24 & {$[\mathrm{Ar}] 3 \mathrm{~d}^{5}$} & ${ }^{6} \mathrm{~S}$ & & & 0.0 & 6 & - \\
\hline Mn I & 1 & 25 & {$[\mathrm{Ar}] 3 \mathrm{~d}^{5} 4 \mathrm{~s}^{2}$} & ${ }^{6} \mathrm{~S}$ & 1667.80 & 59959.40 & 0.0 & 6 & Hyd \\
\hline Mn II & 1 & 25 & {$[\mathrm{Ar}] 3 \mathrm{~d}^{5} 4 \mathrm{~s}$} & ${ }^{7} \mathrm{~S}$ & & & 0.0 & 7 & - \\
\hline $\mathrm{Fe} I$ & 1 & 26 & {$[\mathrm{Ar}] 3 \mathrm{~d}^{6} 4 \mathrm{~s}^{2}$} & ${ }^{5} \mathrm{D}$ & 1589.04 & 63737.00 & 402.96 & 25 & $\mathrm{OP}$ \\
\hline $\mathrm{Fe} I$ & 2 & 26 & {$[\mathrm{Ar}] 3 \mathrm{~d}^{7}\left({ }^{4} \mathrm{~F}\right) 4 \mathrm{~s}$} & ${ }^{5} \mathrm{~F}$ & 1789.67 & & 7457.82 & 35 & OP \\
\hline $\mathrm{Fe} I$ & 3 & 26 & {$[\mathrm{Ar}] 3 \mathrm{~d}^{7}\left({ }^{4} \mathrm{~F}\right) 4 \mathrm{~s}$} & ${ }^{3} \mathrm{~F}$ & $* 1873.48$ & & 12408.10 & 21 & $\mathrm{OP}$ \\
\hline $\mathrm{Fe} I$ & 4 & 26 & {$[\mathrm{Ar}] 3 \mathrm{~d}^{7}\left({ }^{4} \mathrm{P}\right) 4 \mathrm{~s}$} & ${ }^{5} \mathrm{P}$ & 2193.69 & & 17761.40 & 15 & OP \\
\hline $\mathrm{Fe} I$ & 5 & 26 & {$[\mathrm{Ar}] 3 \mathrm{~d}^{6} 4 \mathrm{~s}^{2}$} & ${ }^{3} \mathrm{P}$ & 2266.85 & & 19232.20 & 9 & $\mathrm{OP}$ \\
\hline Fe II & 1 & 26 & {$[\mathrm{Ar}] 3 \mathrm{~d}^{6}\left({ }^{5} \mathrm{D}\right) 4 \mathrm{~s}$} & ${ }^{6} \mathrm{D}$ & & & 0.00 & 30 & - \\
\hline CoI & 1 & 27 & {$[\mathrm{Ar}] 3 \mathrm{~d}^{7} 4 \mathrm{~s}^{2}$} & ${ }^{4} \mathrm{~F}$ & 1616.96 & 63430.0 & 792.80 & 28 & Hyd \\
\hline Co II & 1 & 27 & {$[\mathrm{Ar}] 3 \mathrm{~d}^{8}$} & ${ }^{3} \mathrm{~F}$ & & & 0.0 & 21 & - \\
\hline Ni I & 1 & 28 & {$[\mathrm{Ar}] 3 \mathrm{~d}^{8} 4 \mathrm{~s}^{2}$} & ${ }^{3} \mathrm{~F}$ & 1676.27 & 61600.0 & 971.80 & 21 & Hyd \\
\hline Ni II & 1 & 28 & {$[\mathrm{Ar}] 3 \mathrm{~d}^{9}$} & ${ }^{2} \mathrm{D}$ & & & 0.0 & 10 & - \\
\hline $\mathrm{Cu} \mathrm{I}$ & 1 & 29 & {$[\mathrm{Ar}] 3 \mathrm{~d}^{1} 04 \mathrm{~s}$} & ${ }^{2} S$ & 1604.69 & 62317.44 & 0.0 & 2 & Hyd \\
\hline $\mathrm{Cu}$ II & 1 & 29 & {$[\mathrm{Ar}] 3 \mathrm{~d}^{1} 0$} & ${ }^{1} \mathrm{~S}$ & & & 0.0 & 1 & - \\
\hline Zn I & 1 & 30 & {$[\mathrm{Ar}] 3 \mathrm{~d}^{1} 04 \mathrm{~s}^{2}$} & ${ }^{1} \mathrm{~S}$ & 1319.80 & 75769.33 & 0.0 & 1 & Hyd \\
\hline Zn II & 1 & 30 & {$[\mathrm{Ar}] 3 \mathrm{~d}^{1} 04 \mathrm{~s}$} & ${ }^{2} \mathrm{~S}$ & & & 0.0 & 2 & - \\
\hline
\end{tabular}

${ }^{1}$ John (1988); ${ }^{2}$ Mihalas (1967); ${ }^{3}$ Koester et al. (1985); ${ }^{4}$ hydrogenic treatment; ${ }^{5}$ OPACITY PROJECT and in case of iron the IRON PROJECT. 